\title{
Computing Clebsch-Gordan matrices with applications in elasticity theory
}

\author{
S. Selivanova*
}

\begin{abstract}
We provide an algorithm of computing Clebsch-Gordan coefficients for irreducible representations, with integer weights, of the rotation group $S O(3)$ and demonstrate the convenience of this algorithm for constructing new (to our knowledge) models in anisotropic elasticity theory.
\end{abstract}

Key words: group of rotations, irreducible representations, ClebschGordan matrices, elasticity theory.

\section{Introduction}

The aim of this paper is to provide an algorithm of computing Clebsch-Gordan coefficients, written in a convenient real-valued matrix form, for irreducible representations, with integer weights, of the rotation group $S O(3)$, and to show its convenience for modelling anisotropic elasticity problems.

The Clebsch-Gordan coefficients, arising from Kronecker products of representations (see Section 2 for definitions), are a classical notion in group representation theory, and they are widely used in mathematical physics, see e.g. 2, 4, 14, 17, 21, 23, 24. In [8, 9, 12, 16] it is shown how, written in a matrix form, they allow to construct invariant differential operators (as $\frac{\partial}{\partial x_{i}} G_{1[L, L \pm 1]}^{i}$, where $G_{1[L, L \pm 1]}^{i}$ are particular examples of Clebsch-Gordan matrices, see definitions and examples in the paper) and matrix spherical functions (as products of relevant Clebsch-Gordan matrices and homogeneous polynomials). It helps to write many (systems of) first-order PDEs invariant under rotations in a convenient form and to find explicit solutions of Cauchy problems for them, see e.g. discussions on the examples of elasticity, acoustics and Maxwell equations in the mentioned references.

The algorithm described in Section 4 relies on formulas derived recently in the papers [7, 13. (which were written in continuation of the monograph 9]), where a special basis, w.r.t. which the matrices of the representations are real orthogonal (in contrast to complex unitary matrices in most literature on

${ }^{*}$ This research was partially supported by the Federal Target Grant "Scientific and educational personnel of innovative Russia" for 2009-2013, program 1.2.2, contract No. 8217, and by the Russian Foundation for Basic Research, project No. 12-01-31183. 
mathematical physics), is proposed. The construction of this basis and further technical details are described in Sections 2, 3. The general idea of the algorithm consists of calculating highest-/lowest-weight coefficients first and then applying lowering/raising operators and is well-known (see e.g. [2, 4, 24] and modern improvements [1, 5]). The (probably) new aspect is the special basis of [7, 13, and the spinor technique we use for our computations. In comparison to other recent papers on the subject, the presentation is very elementary, and possibility of application to (linear and nonlinear) elasticity theory is demonstrated (Section 5).

In particular, we use the Clebsch-Gordan matrices to construct quadratic invariants and write down an equation of state of an anisotropic elastic media, as a thermodynamical potential depending on these invariants. By using purely group representation methods we are able to write down the precise form of the invariants for all crystal systems, without any complicated geometric considerations. The parameters of the invariant quadratic forms may be interpreted as the parameters of the media from the Hook's law (Lame coefficients in the isotropic case), in accordance with well-known facts of elasticity theory [19, 15, 22.

This construction can be used to write elasticity equations in an invariant form which is convenient for modelling complicated physical processes like exploding welding of metals and for applying numerical methods (the work in this direction is in progress and will apear in forthcoming publications). These considerations are a development of the paper [20] and are in line with the works [6, 10, 11, 18.

\section{Preliminaries}

Let us first recall some basic notions following the book [9]. The rotations of a three-dimensional Euclidean space $\mathbb{R}^{3}$ constitute the group

$$
S O(3)=\left\{g \in G L(3) \mid g^{T} g=I_{3}, \operatorname{det}(g)=1\right\},
$$

where $G L(3)$ is the group of all nondegenerate $3 \times 3$ matrices, while $I_{3}$ is the unit matrix.

It is said that we have a representation $T_{G}$ of a group $G$ in a $k$-dimensional vector space $\mathcal{L}$, if to each element $g \in G$ there corresponds a linear mapping $T_{g}: \mathcal{L} \rightarrow \mathcal{L}$ such that

$$
T_{e}=I_{k}, \quad T_{g_{1} \cdot g_{2}}=T_{g_{1}} \cdot T_{g_{2}},
$$

where $e$ is the identity of the group $G$.

The representation $T_{G}$ is called irreducible, if in $\mathcal{L}$ there are no nontrivial subspaces invariant under all mappings $T_{g}$, where $g \in G$. The number $N$ (integer or half of an integer), such that $k=2 N+1$, is called the weight of the irreducible representation. It is well-known that single-valued irreducible representations of $S O(3)$ can exist only in odd-dimensional spaces (i.e. when $N$ is integer), moreover, for a fixed number $N$, the corresponding representation is unique up to equivalence. In this paper we consider only integer weights $N$. 
The elements $\{g(t) \in G\}_{t \in \mathbb{R}}$ constitute a one-parameter subgroup of the group $G$, if

$$
g(t+s)=g(t) \cdot g(s) \text { and } g(0)=e .
$$

Let $\mathcal{L}_{N}$ be the $(2 N+1)$-dimensional vector space in which the action of an irreducible representation $T_{G}$ of $G=S O(3)$ is defined, and let us denote the Cartesian coordinates in $\mathbb{R}^{3}$ as $x_{-1}, x_{0}, x_{1}$. Then the one-parameter subgroups corresponding to rotations w.r.t. these axes are described by the families of matrices

$$
\begin{gathered}
g_{1}(\omega)=\left[\begin{array}{ccc}
1 & 0 & 0 \\
0 & \cos (\omega) & -\sin (\omega) \\
0 & \sin (\omega) & \cos (\omega)
\end{array}\right], g_{-1}(\omega)=\left[\begin{array}{ccc}
\cos (\omega) & 0 & \sin (\omega) \\
0 & 1 & 0 \\
-\sin (\omega) & 0 & \cos (\omega)
\end{array}\right], \\
g_{0}(\omega)=\left[\begin{array}{ccc}
\cos (\omega) & -\sin (\omega) & 0 \\
\sin (\omega) & \cos (\omega) & 0 \\
0 & 0 & 1
\end{array}\right],
\end{gathered}
$$

where $\omega \in \mathbb{R}$.

Denote as $J_{j}, j=-1,0,1$, the infinitesimal operators of the representations of these subgroups:

$$
J_{j}=\left.\frac{d}{d \omega} T_{g_{j}(\omega)}\right|_{\omega=0} .
$$

There are different possibilities to chose a canonical, w.r.t. the representation $T_{G}$, basis in $\mathcal{L}_{N}$. A standard way, see e.g. 4, 9, 24, is to chose vectors on which the infinitesimal operators act as follows

$$
\begin{gathered}
i J_{0} e_{N}^{n}=n e_{N}^{n}, n=-N, \ldots, N, \\
\left(i J_{1}+J_{-1}\right) e_{N}^{n}= \begin{cases}-\sqrt{(N-n+1)(N+n)} e_{N}^{n-1}, & n=-N+1, \ldots, N, \\
0, & n=-N\end{cases} \\
\left(i J_{1}-J_{-1}\right) e_{N}^{n}= \begin{cases}-\sqrt{(N-n)(N+n+1)} e_{N}^{n+1}, & n=-N, \ldots, N-1, \\
0, & n=N .\end{cases}
\end{gathered}
$$

Note that $e_{N}^{n}$ are eigenvectors of the operator $J_{0}$ (they are defined up to a multiplicative constant), while formulas (5) and (6) allow to compute the basis vectors recursively. W. r. t. such basis, which is called a canonical basis of "e" type, $T_{G}$ is defined by unitary matrices of dimension $(2 N+1) \times(2 N+1)$.

In $\left[7\right.$ a basis, w.r.t. to which $T_{G}$ is defined by (real) orthogonal matrices, is introduced, and it is called a canonical basis of " $h$ " type. This basis is related to the previous one by means of a unitary transformation:

$$
\left\{\begin{array}{l}
h_{N}^{-n}=\frac{(-i)^{N-1}}{\sqrt{2}}\left[(-1)^{n} e_{N}^{n}-e_{N}^{-n}\right], \\
h_{N}^{0}=(-i)^{N} e_{N}^{0}, \\
h_{N}^{n}=\frac{-(-i)^{N}}{\sqrt{2}}\left[(-1)^{n} e_{N}^{n}+e_{N}^{-n}\right] .
\end{array}\right.
$$


The action of the infinitesimal operators on this basis is as follows:

$$
\begin{gathered}
\left(\begin{array}{cc}
0 & J_{0} \\
-J_{0} & 0
\end{array}\right)\left(\begin{array}{c}
h_{N}^{-n} \\
h_{N}^{n}
\end{array}\right)=n\left(\begin{array}{c}
h_{N}^{-n} \\
h_{N}^{n}
\end{array}\right), n \geq 0 \\
\left(\begin{array}{cc}
J_{-1} & J_{1} \\
-J_{1} & J_{-1}
\end{array}\right)\left(\begin{array}{c}
h_{N}^{-n} \\
h_{N}^{n}
\end{array}\right)=\sqrt{(N+n)(N-n+1)}\left(\begin{array}{c}
h_{N}^{-n+1} \\
h_{N}^{n-1}
\end{array}\right), n \geq 2, \\
\left(\begin{array}{cc}
J_{-1} & J_{1} \\
-J_{1} & J_{-1}
\end{array}\right)\left(\begin{array}{c}
h_{N}^{-1} \\
h_{N}^{1}
\end{array}\right)=-\sqrt{2 N(N+1)}\left(\begin{array}{c}
0 \\
h_{N}^{0}
\end{array}\right), \\
\left\{\begin{array}{cc}
\left(\begin{array}{cc}
J_{-1} & -J_{1} \\
J_{1} & J_{-1}
\end{array}\right)\left(\begin{array}{c}
h_{N}^{-n} \\
h_{N}^{n}
\end{array}\right)=-\sqrt{(N+n+1)(N-n)}\left(\begin{array}{c}
h_{N}^{-n-1} \\
h_{N}^{n+1}
\end{array}\right), n \geq 1, \\
\left(\begin{array}{cc}
J_{-1} & J_{1} \\
J_{1} & J_{-1}
\end{array}\right)\left(\begin{array}{c}
0 \\
h_{N}^{0}
\end{array}\right)=\sqrt{\frac{N(N+1)}{2}}\left(\begin{array}{c}
h_{N}^{-1} \\
h_{N}^{1}
\end{array}\right),
\end{array}\right.
\end{gathered}
$$

Recall that a Kronecker product $T_{g}=T_{g}^{1} \times T_{g}^{2}$ of the representation $T_{g}^{1}$ of weight $N_{1}$ and $T_{g}^{2}$ of weight $N_{2}$ is a representation which acts on a matrix $B$ of dimension $\left(2 N_{1}+1\right) \times\left(2 N_{2}+1\right)$ as

$$
T_{g} B=T_{g}^{1} B\left(T_{g}^{2}\right)^{T} .
$$

The definition of the Clebsch-Gordan matrices, that we are going to compute, arises from the following statement [7].

Theorem. If the representations $T_{g}^{1}$ and $T_{g}^{2}$ are irreducible, then $T_{g}^{1} \times T_{g}^{2}$ can be decomposed into a direct sum of irreducible representations of the following weights:

$$
N=\left|N_{1}-N_{2}\right|,\left|N_{1}-N_{2}\right|+1, \ldots, N_{1}+N_{2} .
$$

Such decomposition is realized by means of the Clebsch-Gordan matrices, which constitute canonical bases of the corresponding spaces of matrices:

$$
G_{N\left[N_{1}, N_{2}\right]}^{n} \text { of dimension }\left(2 N_{1}+1\right) \times\left(2 N_{2}+1\right), n=-N, N+1, \ldots, N .
$$

These matrices are real-valued, orthonormal:

$$
\operatorname{tr}\left\{\left(G_{N\left[N_{1}, N_{2}\right]}^{n}\right)^{T} G_{N\left[N_{1}, N_{2}\right]}^{m}\right\}=\delta_{m n} \text { (the Kronecker symbol), }
$$

possess one or two non-zero diagonals, and satisfy the following symmetry property:

$$
G_{N\left[N_{1}, N_{2}\right]}^{n}=(-1)^{N+N_{1}+N_{2}}\left(G_{N\left[N_{2}, N_{1}\right]}^{n}\right)^{T} .
$$

For computations, different realizations of the irreducible representations are useful, in particular: in the space of homogeneous polynomials of three real variables; in the space of homogeneous spinor polynomials of two complex variables; in the space of matrices. Our computations rely on the last two ones; we describe them in the next section. 


\section{Different realizations of irreducible represen- tations of the rotation group}

A spinor polynomial is a homogeneous polynomial $f(\xi, \eta)$ of degree $2 N$ of two complex variables $\xi, \eta$. The dimension of this space is equal to $2 N+1$.

As it is known, the elements of $S O(3)$ can be parameterized by unitary matrices $g \in S U(2)$ in such a way that to any irreducible representation of $S U(2)$ of an integer weight $N$ there corresponds a unique representation of $S O(3)$ in a space of odd dimension equal to $2 N+1$. This parametrization helps to describe all single-valued irreducible representations of $S O(3)$, since it is sometimes more convenient to make computations for $S U(2)$. For simplicity of notation we denote the one-parameter subgroups of $S U(2)$ and the corresponding infinitesimal operators in the same way $g_{j}(w)$ and $J_{j}$, as for $S O(3)$ above.

In the space of spinor polynomials the irreducible representation of $S U(2)$ of weight $N$ is realized by the following formula [7]: for

$$
g \in S U(2), g=\left(\begin{array}{cc}
\alpha & \beta \\
-\bar{\beta} & \bar{\alpha}
\end{array}\right)
$$

we have

$$
T_{g} f(\xi, \eta)=f\left(\xi^{\prime}, \eta^{\prime}\right) \equiv f(\bar{\alpha} \xi-\beta \eta, \bar{\beta} \xi+\alpha \eta) .
$$

The canonical basis of "e" type consists of the polynomials

$$
e_{N}^{n}(\xi, \eta)=\tilde{\rho}(N, n) \xi^{N+n} \eta^{N-n}=(-1)^{N+n} \sqrt{\frac{(2 N+1) !}{(N+n) !(N-n) !}} \xi^{N+n} \eta^{N-n},
$$

where $n=-N, \ldots N$. According to (7), the canonical basis of "h" type is defined as

$$
\begin{aligned}
& h_{N}=e_{N} U_{N}, \\
& e_{N}=h_{N} V_{N},
\end{aligned}
$$

where

$$
\begin{aligned}
& h_{N}=\left(h_{N}^{-N}, h_{N}^{-N+1}, \ldots, h_{N}^{0}, \ldots, h_{N}^{N}\right), e_{N}^{\cdot}=\left(e_{N}^{-N}, e_{N}^{-N+1}, \ldots, e_{N}^{0}, \ldots, e_{N}^{N}\right),
\end{aligned}
$$

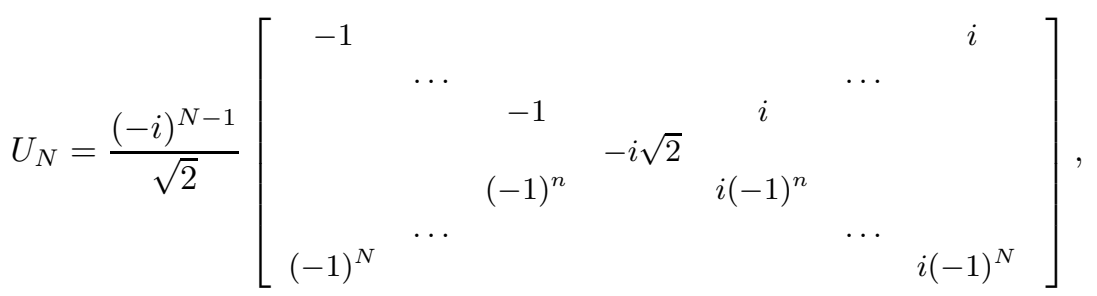




$$
\begin{aligned}
& U_{N}^{*} U_{N}=I_{2 N+1} \text { and }
\end{aligned}
$$

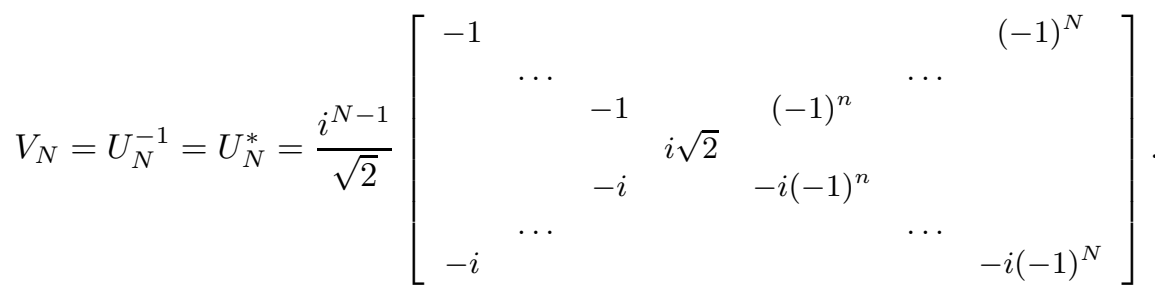

The product of two irreducible representations of weights $N_{1}$ and $N_{2}$ (of dimensions $2 N_{1}+1$ and $2 N_{2}+1$ ), can be realized in the space of bispinor polynomials $f\left(\xi_{1}, \eta_{1} ; \xi_{2}, \eta_{2}\right)$, homogeneous of degrees $2 N_{1}$ and $2 N_{2}$ on $\xi_{1}, \eta_{1}$ and $\xi_{2}, \eta_{2}$, respectively:

$$
T_{g} f\left(\xi_{1}, \eta_{1} ; \xi_{2}, \eta_{2}\right)=f\left(\bar{\alpha} \xi_{1}-\beta \eta_{1}, \bar{\beta} \xi_{1}+\alpha \eta_{1} ; \bar{\alpha} \xi_{2}-\beta \eta_{2}, \bar{\beta} \xi_{2}+\alpha \eta_{2}\right) .
$$

This representation acting in a $\left(2 N_{1}+1\right) \cdot\left(2 N_{2}+1\right)$-dimensional space is reducible and it can be decomposed into irreducible ones as in (12). The canonical bases of "e" type look as 9 , 8,

$$
\begin{gathered}
\mu_{N\left[N_{1}, N_{2}\right]}^{n}\left(\xi_{1}, \eta_{1} ; \xi_{2}, \eta_{2}\right)= \\
=\hat{\rho}\left(N, N_{1}, N_{2}\right)\left(\xi_{1} \eta_{2}-\eta_{1} \xi_{2}\right)^{N_{1}+N_{2}-N}\left(-\xi_{2} \frac{\partial}{\partial \xi_{1}}-\eta_{2} \frac{\partial}{\partial \eta_{1}}\right)^{N-N_{1}+N_{2}} e_{N}^{n}\left(\xi_{1}, \eta_{1}\right),
\end{gathered}
$$

where $n=-N,-N+1, \ldots, N$, and $N_{1}, N_{2}$ are fixed and $N$ is as in (12);

$$
\hat{\rho}\left(N, N_{1}, N_{2}\right)=\sqrt{\frac{\left(2 N_{1}+1\right) !\left(2 N_{2}+1\right) !\left(N+N_{1}-N_{2}\right) !}{2 N !\left(N_{1}+N_{2}-N\right) !\left(N-N_{1}+N_{2}\right) !\left(N+N_{1}+N_{2}+1\right) !}} .
$$

The complete proof of this fact can be found in [9] (Section 13). Since this book is in Russian, we recall briefly its main steps.

- First, the infinitesimal operators acting in the space of bispinor polynomials are calculated (for spinor polynomials it is a straightforward calculation by definition, and for the bispinor case the definition of the Kronecker product of representations is used). The answer is as follows:

$$
\begin{aligned}
\left(i J_{1}+J_{-1}\right) & =\eta_{1} \frac{\partial}{\partial \xi_{1}}+\eta_{2} \frac{\partial}{\partial \xi_{2}}, \quad\left(i J_{1}-J_{-1}\right)=\xi_{1} \frac{\partial}{\partial \eta_{1}}+\xi_{2} \frac{\partial}{\partial \eta_{2}} \\
i J_{0} & =\frac{1}{2}\left(\xi_{1} \frac{\partial}{\partial \xi_{1}}-\eta_{1} \frac{\partial}{\partial \eta_{1}}\right)+\frac{1}{2}\left(\xi_{2} \frac{\partial}{\partial \xi_{2}}-\eta_{2} \frac{\partial}{\partial \eta_{2}}\right) .
\end{aligned}
$$

- It is noticed (by direct computation of the action of the infinitesimal operators) that the products $e_{N_{1}}^{n_{1}} e_{N_{2}}^{n_{2}}$ do not constitute a canonical basis in the space of bispinor polynomials. The form of the polynomials needed is "guessed" by taking in account the identities

$$
\left(i J_{1}+J_{-1}\right)\left(\xi_{1} \eta_{2}-\xi_{2} \eta_{1}\right)^{q}=0, \quad\left(i J_{1}-J_{-1}\right)\left(\xi_{1} \eta_{2}-\xi_{2} \eta_{1}\right)^{q}=0
$$


and by choosing a proper norming after calculating the scalar square of the polynomial

$$
\left(\xi_{q} \eta_{2}-\xi_{2} \eta_{1}\right)^{a} \eta_{1}^{b} \eta_{2}^{c}
$$

- Finally, a direct substitution of the basis (22) into the equalities (4)-(5) shows that it satisfies the definition of a canonical basis of "e" type.

Further, according to (7), the "h" type bases look as

$$
\begin{gathered}
\nu_{N\left[N_{1}, N_{2}\right]}^{n}\left(\xi_{1}, \eta_{1} ; \xi_{2}, \eta_{2}\right)=\hat{\rho}\left(N, N_{1}, N_{2}\right)\left(\xi_{1} \eta_{2}-\eta_{1} \xi_{2}\right)^{N_{1}+N_{2}-N} . \\
\cdot\left(-\xi_{2} \frac{\partial}{\partial \xi_{1}}-\eta_{2} \frac{\partial}{\partial \eta_{1}}\right)^{N-N_{1}+N_{2}} h_{N}^{n}\left(\xi_{1}, \eta_{1}\right) .
\end{gathered}
$$

These bases are related as

$$
\mu_{N\left[N_{1}, N_{2}\right]}=\nu_{N\left[N_{1}, N_{2}\right]}^{\cdot} V_{N} .
$$

The Clebsch-Gordan matrices $C_{N\left[N_{1}, N_{2}\right]}^{n}$ and $G_{N\left[N_{1}, N_{2}\right]}^{n}$ are introduced as the coefficients of decomposition of the basis polynomials $\mu_{N\left[N_{1}, N_{2}\right]}^{n}$ and $\nu_{N\left[N_{1}, N_{2}\right]}^{n}$ by the polynomials $\left\{e_{N_{1}}^{n_{1}} e_{N_{2}}^{n_{2}}\right\}$ and $\left\{h_{N_{1}}^{n_{1}} h_{N_{2}}^{n_{2}}\right\}$, respectively:

$$
\begin{aligned}
& \mu_{N\left[N_{1}, N_{2}\right]}^{n}=\dot{e}_{N_{1}} C_{N\left[N_{1}, N_{2}\right]}^{n}\left(\dot{e}_{N_{2}}\right)^{T}, \\
& \nu_{N\left[N_{1}, N_{2}\right]}^{n}=h_{N_{1}} G_{N\left[N_{1}, N_{2}\right]}^{n}\left(h_{N_{2}}\right)^{T}
\end{aligned}
$$

It is easy to see [7], that the matrices $C_{N\left[N_{1}, N_{2}\right]}^{n}, n=-N, \ldots, N$ and $G_{N\left[N_{1}, N_{2}\right]}^{n}, \quad n=-N, \ldots, N$ constitute canonical bases of type "e" and of type "h", respectively, in the subspace of $\left(2 N_{1}+1\right) \times\left(2 N_{2}+1\right)$-dimensional matrices, where an irreducible representation of dimension $N, N=\left|N_{1}-N_{2}\right|, \ldots, N_{1}+N_{2}$ is acting.

The following formula [7, which is a corollary of the formulas (22), (29), will be useful for computations of the nonzero Clebsch-Gordan coefficients in the next section. For all $n \in\{-N,-N+1, \ldots, N\}, n_{2} \in\left\{-N_{2},-N_{2}+1, \ldots, N_{2}\right\}$ we have

$$
\begin{gathered}
\left\{\rho\left(N, N_{1}, N_{2}\right)\left(\frac{\partial}{\partial \xi_{0}} \frac{\partial}{\partial \eta}-\frac{\partial}{\partial \eta_{0}} \frac{\partial}{\partial \xi}\right)^{N-N_{1}+N_{2}} e_{N}^{n}\left(\xi_{0}, \eta_{0}\right)\right\}||_{\xi_{0}=\xi, \eta_{0}=\eta} \\
\cdot(-1)^{N_{2}-n_{2}} e_{N_{2}}^{-n_{2}}(\xi, \eta)=\sum_{n_{1}} c_{N\left[N_{1}, N_{2}\right]}^{n\left[n_{1}, n_{2}\right]} e_{N_{1}}^{n_{1}}(\xi, \eta)
\end{gathered}
$$

where

$$
\rho\left(N, N_{1}, N_{2}\right)=\sqrt{\frac{\left(2 N_{1}+1\right) !\left(N_{1}+N_{2}-N\right) !\left(N+N_{1}-N_{2}\right) !}{(2 N) !\left(2 N_{2}+1\right) !\left(N+N_{1}+N_{2}+1\right) !\left(N-N_{1}+N_{2}\right) !}} .
$$




\section{Computing the Clebsch-Gordan matrices}

We want to compute the nonzero elements of the matrices

$$
G_{N\left[N_{1}, N_{2}\right]}^{ \pm n}, n=0, \ldots, N, N=\left|N_{1}-N_{2}\right|,\left|N_{1}-N_{2}\right|+1, \ldots, N_{1}+N_{2}
$$

for arbitrary integer weights $N_{1}, N_{2}$. Note that in a similar way we can simply obtain the matrices $C_{N\left[N_{1}, N_{2}\right]}^{ \pm n}$.

The general scheme of computation, which we present in more details below, is as follows:

1) Compute the nonzero diagonal of the two matrices $C_{N\left[N_{1}, N_{2}\right]}^{ \pm N}$, by means of (31).

2) Compute the two matrices $G_{N\left[N_{1}, N_{2}\right]}^{ \pm N}$ by means of the transformation formulas (20) and (28).

3) Compute other matrices by means of recurrent formulas (9), (10), which allow to define $G_{N\left[N_{1}, N_{2}\right]}^{ \pm(n-1)}$ from $G_{N\left[N_{1}, N_{2}\right]}^{ \pm n}$.

Now let us describe these three stages more precisely.

- To carry out the first stage we use the well-known property of the ClebschGordan coefficients stating that

$$
c_{N\left[N_{1}, N_{2}\right]}^{n\left[n_{1}, n_{2}\right]}=0 \text { for } n_{1}+n_{2} \neq n
$$

i.e. each matrix $C_{N\left[N_{1}, N_{2}\right]}^{n}$ possesses only one nonzero diagonal, thus the sum in the right-hand part of (31), for $n=N$, consists of only one nonzero summand (when $n_{1}=n-n_{2}$ ).

More precisely, for $n=N$ the elements of the nonzero diagonal, have the numbers $(N-k, k)$, where $k=N-N_{1}, N-N_{1}+1, \ldots, N_{2}$. Rewriting (31) and taking into account (32) and (18), we obtain

$$
\begin{gathered}
c_{N\left[N_{1}, N_{2}\right]}^{N[N-k, k]}=(-1)^{N_{2}-k} \rho\left(N, N_{1}, N_{2}\right) \frac{\tilde{\rho}\left(N_{2},-k\right)}{\tilde{\rho}\left(N_{1}, N-k\right)} \sqrt{2 N+1} \cdot \\
\cdot\left[\left(N+N_{1}-N_{2}+1\right) \ldots(2 N-1)(2 N)\right] \cdot\left[\left(N_{1}-N+k+1\right) \ldots\left(N_{2}+k-1\right)\left(N_{2}+k\right)\right] .
\end{gathered}
$$

For $n=-N$, the elements of the nonzero diagonal have numbers $(-N-$ $k, k), \quad k=-N_{2},-N_{2}+1, \ldots, N_{1}-N$, and we obtain

$$
\begin{gathered}
c_{N\left[N_{1}, N_{2}\right]}^{-N[-N-k]}=(-1)^{N_{2}-k} \rho\left(N, N_{1}, N_{2}\right) \frac{\tilde{\rho}\left(N_{2},-k\right)}{\tilde{\rho}\left(N_{1},-N-k\right)} \sqrt{2 N+1} \cdot \\
\cdot\left[\left(N+N_{1}-N_{2}+1\right) \ldots(2 N-1)(2 N)\right] \cdot\left[\left(N_{1}-N-k+1\right) \ldots\left(N_{2}-k-1\right)\left(N_{2}-k\right)\right] .
\end{gathered}
$$

The case $N=0$ (and $N_{2}=N_{1}$ ) is considered independently:

$$
c_{0\left[N_{1}, N_{1}\right]}^{0[-k, k]}=\frac{(-1)^{N_{1}-k}}{\sqrt{2 N_{1}+1}}, k=-N_{1},-N_{1}+1, \ldots, N_{1} \text {. }
$$


- Now turn to the second stage. From the formulas (28) and (29) we have

$$
\begin{gathered}
\nu_{N\left[N_{1}, N_{2}\right]}^{N}=\frac{-(-i)^{N}}{\sqrt{2}}\left[(-1)^{N} \mu_{N\left[N_{1}, N_{2}\right]}^{N}+\mu_{N\left[N_{1}, N_{2}\right]}^{-N}\right]= \\
=\frac{-(-i)^{N}}{\sqrt{2}}\left[(-1)^{N} e_{N_{1}}^{\cdot} C_{N\left[N_{1}, N_{2}\right]}^{N}\left(e_{N_{2}}^{\cdot}\right)^{T}+e_{N_{1}}^{\cdot} C_{N\left[N_{1}, N_{2}\right]}^{-N}\left(e_{N_{2}}^{\cdot}\right)^{T}\right]= \\
=\frac{-(-i)^{N}}{\sqrt{2}} h_{N_{1}} V_{N_{1}}\left[(-1)^{N} C_{N\left[N_{1}, N_{2}\right]}+C_{N\left[N_{1}, N_{2}\right]}^{-N}\right]\left(V_{N_{2}}\right)^{T}\left(h_{N_{2}}\right)^{T},
\end{gathered}
$$

hence

$$
G_{N\left[N_{1}, N_{2}\right]}^{N}=\frac{-(-i)^{N}}{\sqrt{2}} V_{N_{1}}\left\{(-1)^{N} C_{N\left[N_{1}, N_{2}\right]}^{N}+C_{N\left[N_{1}, N_{2}\right]}^{-N}\right\}\left(V_{N_{2}}\right)^{T} .
$$

In a similar way,

$$
G_{N\left[N_{1}, N_{2}\right]}^{-N}=\frac{(-i)^{N-1}}{\sqrt{2}} V_{N_{1}}\left\{(-1)^{N} C_{N\left[N_{1}, N_{2}\right]}^{N}-C_{N\left[N_{1}, N_{2}\right]}^{-N}\right\}\left(V_{N_{2}}\right)^{T}
$$

For $N=0\left(N_{2}=N_{1}\right)$, we have

$$
G_{0\left[N_{1}, N_{1}\right]}^{0}=V_{N_{1}} C_{0\left[N_{1}, N_{1}\right]}^{0}\left(V_{N_{1}}\right)^{T} .
$$

Note that the matrices calculated on this stage are real-valued.

- Finally, in order to compute other matrices $G_{N\left[N_{1}, N 2\right]}^{ \pm n}, n<N$, on the third stage of the algorithm, we need to compute first the action of the infinitesimal operators $J_{ \pm 1}$, see (3), (9), (10).

By definition,

$$
J_{n}=\left.\frac{d}{d \omega} T_{g_{n}(\omega)}\right|_{\omega=0}, \quad n=-1,0,1,
$$

where $g_{n}(\omega)$ are one-parameter subgroups of $S U(2)$.

As already mentioned in the previous section, in the space of spinor polynomials, $J_{ \pm 1}$ are given by formulas

$$
J_{-1}=\frac{1}{2}\left(-\eta \frac{\partial}{\partial \xi}+\xi \frac{\partial}{\partial \eta}\right), J_{1}=\frac{i}{2}\left(\eta \frac{\partial}{\partial \xi}+\xi \frac{\partial}{\partial \eta}\right)
$$

From particular cases of the formula (31), written in the basis "h", it follows that

$$
\frac{(-1)^{n} \sqrt{3} J_{n}}{\sqrt{N(N+1)(2 N+1)}} h_{N}^{k}(\xi, \eta)=\sum_{j} g_{1[N, N]}^{n[j, k]} h_{N}^{j}(\xi, \eta),
$$

and hence

$$
J_{ \pm 1}^{N}=-\sqrt{\frac{N(N+1)(2 N+1)}{3}} G_{1[N, N]}^{ \pm 1}
$$


where by $J_{ \pm 1}^{N}$ we mean the corresponding infinitesimal operators of the representations of weight $N$. This formulas are constructive, since the matrices $G_{1[N, N]}^{ \pm 1}$ were computed on the first stage.

Let us again consider the space of $\left(2 N_{1}+1\right) \times\left(2 N_{2}+1\right)$ matrices, which is isomorphic to the space of bispinor polynomials and denote there the infinitesimal operators as $J_{ \pm 1}^{N_{1} \times N_{2}}$. Recall that in this space the Clebsch-Gordan matrices

$$
G_{N\left[N_{1}, N_{2}\right]}^{ \pm n}, n=0, \ldots, N, N=\left|N_{1}-N_{2}\right|,\left|N_{1}-N_{2}\right|+1, \ldots, N_{1}+N_{2}
$$

constitute a canonical basis of " $\mathrm{h}$ " type.

The action of the operator $J_{ \pm 1}^{N_{1} \times N_{2}}$ on the matrix $B$ of dimension $\left(2 N_{1}+\right.$ 1) $\times\left(2 N_{2}+1\right)$ is computed as

$$
\begin{gathered}
J_{ \pm 1}^{N_{1} \times N_{2}} B=-\frac{1}{\sqrt{3}}\left\{\sqrt{N_{1}\left(N_{1}+1\right)\left(2 N_{1}+1\right)} G_{1\left[N_{1}, N_{1}\right]}^{ \pm 1} B+\right. \\
\left.\sqrt{N_{2}\left(N_{2}+1\right)\left(2 N_{2}+1\right)} B\left(G_{1\left[N_{2}, N_{2}\right]}^{ \pm 1}\right)^{T}\right\} .
\end{gathered}
$$

It follows from the definition of the Kronecker product and the infinitesimal operator and taking into account the formula (44):

$$
\begin{gathered}
\left(T_{g}^{1} \times T_{g}^{2}\right) B=T_{g}^{1} B\left(T_{g}^{2}\right)^{T} \\
J_{ \pm 1}^{N_{1} \times N_{2}} B=\left.\frac{d}{d \omega}\left(T_{g_{ \pm 1}(\omega)}^{1} \times T_{g_{ \pm 1}(\omega)}^{1}\right)\right|_{\omega=0} B=J_{ \pm 1}^{N_{1}} B+B J_{ \pm 1}^{N_{2}} .
\end{gathered}
$$

Finally, the recurrent formulas (9) and (10) allow to compute the matrices $G_{N\left[N_{1}, N_{2}\right]}^{ \pm(n-1)}$ from $G_{N\left[N_{1}, N_{2}\right]}^{ \pm n}$.

More precisely, for $N \geq n \geq 2$ we have

$$
\begin{gathered}
G_{N\left[N_{1}, N_{2}\right]}^{-(n-1)}=-\frac{1}{\sqrt{(N+n)(N-n+1)}}\left\{J_{-1}^{N_{1} \times N_{2}} G_{N\left[N_{1}, N_{2}\right]}^{-n}+\right. \\
\left.\quad+J_{1}^{N_{1} \times N_{2}} G_{N\left[N_{1}, N_{2}\right]}^{n}\right\}, \\
G_{N\left[N_{1}, N_{2}\right]}^{(n-1)}=-\frac{1}{\sqrt{(N+n)(N-n+1)}}\left\{-J_{1}^{N_{1} \times N_{2}} G_{N\left[N_{1}, N_{2}\right]}^{-n}+\right. \\
\left.\quad+J_{-1}^{N_{1} \times N_{2}} G_{N\left[N_{1}, N_{2}\right]}^{n}\right\} .
\end{gathered}
$$

For $n=1$ we have

$$
G_{N\left[N_{1}, N_{2}\right]}^{0}=\frac{1}{\sqrt{2 N(N+1)}}\left\{-J_{1}^{N_{1} \times N_{2}} G_{N\left[N_{1}, N_{2}\right]}^{-1}+J_{-1}^{N_{1} \times N_{2}} G_{N\left[N_{1}, N_{2}\right]}^{1}\right\} .
$$

Note also that we can compute the Clebsch-Gordan matrices by this algorithm without the spinor technique (by using only the matrix approach), computing at the first stage the eigenvectors of the infinitesimal operator $J_{0}$ acting in the space of matrices. 


\section{Applications to elasticity theory}

\subsection{Statement of the problem}

Let $T=\left\|s_{i j}\right\|$ (where $i, j=-1,0,1$ ) be a tensor related to the elastic media, e.g. in [20] we consider the Piola-Kirchhoff stress tensor (which is not necessarily symmetric) in Lagrangian coordinates $\xi_{-1}, \xi_{0}, \xi_{1}$, but we also may consider the symmetric Cauchy stress tensor in Eulerian coordinates $x_{-1}, x_{0}, x_{1}$, or some relevant function of the distorsion matrix $C=\left\|c_{i j}\right\|=\left\|\frac{\partial x_{i}}{\partial \xi_{j}}\right\|$, whatever is more convenient for a particular problem. Here we assume for simplicity this tensor be symmetric: $T=T^{*}$, and use, when considering different crystal systems, a technique a bit different from the one used in [20].

The internal energy of the considered media can then be written as a function of $\operatorname{det}(T)$ and five quadratic invariants, which will be constructed below:

$$
H=H\left(\operatorname{det}(T), J_{0}, J_{1}, I_{0}, I_{1}, I_{2}\right) .
$$

For linear elasticity theory, the internal energy is constructed just as the sum of the quadratic invariants.

The symmetric tensor $T$ can be written as

$$
T=\left[\begin{array}{lll}
s_{11} & s_{12} & s_{13} \\
s_{21} & s_{22} & s_{23} \\
s_{31} & s_{32} & s_{33}
\end{array}\right]=p I_{3}+S
$$

Here $p$ is the "pressure", a scalar, i.e. it is transformed by an irreducible representation of weight 0 ;

$S=S^{*}, \operatorname{tr}(S)=0$ is the "deviator" matrix, consisting of five independent elements, which form a vector transformed by an irreducible representation of weight 2 ;

$I_{3}$ is the identity matrix.

The matrix $S$ can be rewritten by means of the corresponding ClebschGordan matrices (which are a canonical basis in the space of symmetric $3 \times 3$ matrices with zero trace) as follows:

$$
\begin{gathered}
S=\left[\begin{array}{ccc}
s_{11}-p & \frac{s_{12}+s_{21}}{2} & \frac{s_{13}+s_{31}}{2} \\
\frac{s_{12}+s_{21}}{2} & s_{22}-p & \frac{s_{23}+s_{32}}{2} \\
\frac{s_{13}+s_{31}}{2} & \frac{s_{23}+s_{32}}{2} & s_{33}-p
\end{array}\right]= \\
=s_{-2} G_{2[1,1]}^{-2}+s_{-1} G_{2[1,1]}^{-1}+s_{0} G_{2[1,1]}^{0}+s_{1} G_{2[1,1]}^{1}+s_{2} G_{2[1,1]}^{2} .
\end{gathered}
$$


Let us denote

$$
\mathbf{s}=\left(\begin{array}{c}
s_{-2} \\
s_{-1} \\
s_{0} \\
s_{1} \\
s_{2}
\end{array}\right)=\left(\begin{array}{c}
-\frac{s_{13}+s_{31}}{\sqrt{2}} \\
\frac{s_{12}+s_{21}}{\sqrt{2}} \\
\frac{\sqrt{3}}{\sqrt{2}}\left(s_{22}-p\right) \\
\frac{s_{23}+s_{32}}{\sqrt{2}} \\
\frac{s_{11}-s_{33}}{\sqrt{2}}
\end{array}\right) .
$$

This vector is transformed by weight 2 , and the corresponding Clebsch-Gordan matrices (calculated by the algorithm from the previous subsection) look as follows:

$$
\begin{gathered}
G_{2[1,1]}^{-2}=\left[\begin{array}{ccc}
0 & 0 & -\frac{1}{\sqrt{2}} \\
0 & 0 & 0 \\
-\frac{1}{\sqrt{2}} & 0 & 0
\end{array}\right], G_{2[1,1]}^{-1}=\left[\begin{array}{ccc}
0 & \frac{1}{\sqrt{2}} & 0 \\
\frac{1}{\sqrt{2}} & 0 & 0 \\
0 & 0 & 0
\end{array}\right], \\
G_{2[1,1]}^{0}=\left[\begin{array}{ccc}
-\frac{1}{\sqrt{6}} & 0 & 0 \\
0 & \frac{2}{\sqrt{6}} & 0 \\
0 & 0 & -\frac{1}{\sqrt{6}}
\end{array}\right], \\
G_{2[1,1]}^{1}=\left[\begin{array}{ccc}
0 & 0 & 0 \\
0 & 0 & \frac{1}{\sqrt{2}} \\
0 & \frac{1}{\sqrt{2}} & 0
\end{array}\right], G_{2[1,1]}^{2}=\left[\begin{array}{ccc}
\frac{1}{\sqrt{2}} & 0 & 0 \\
0 & 0 & 0 \\
0 & 0 & -\frac{1}{\sqrt{2}}
\end{array}\right] .
\end{gathered}
$$

Let us calculate the matrix of the irreducible representation of $S O(3)$ acting in $\mathbb{R}^{5}$ as

$$
\tilde{\mathbf{s}}=A \mathbf{s}
$$

which transforms the above introduced vector

$$
\mathbf{s}=\left(s_{-2}, s_{-1}, s_{0}, s_{1}, s_{2}\right)^{T}
$$

(cf. 13, 4, 24, etc. where the matrix entries of irreducible are calculated with different methods for the general case). The matrix $A$ can be represented as the product $A_{\theta} A_{\varphi} A_{\psi}$ of the matrices of representations of the rotations $U_{\theta}, U_{\varphi}, U_{\psi}$ around the coordinate axes (as it is well-known, any element $U$ of $S O(3)$ is a combination of such rotations).

The transformation

$$
\mathbf{x}^{\prime}=U \mathbf{x}
$$

of the space of vectors

$$
\mathbf{x}=\left(x_{-1}, x_{0}, x_{1}\right)^{T}
$$


induces the transformation $U^{T} S U$ of the tensor $S$, thus in order to calculate the elements $A_{i j}$ of the desired matrix $A$ we first calculate independently each matrix $U^{T} G_{2[1,1]}^{j} U$ of the sum

$$
\tilde{S}=\sum_{j=-2}^{2} s_{j} U^{T} G_{2[1,1]}^{j} U=\sum_{i=-2}^{2} \tilde{s}_{i} G_{2[1,1]}^{i}
$$

and deduce from (58)

$$
\tilde{s}_{i}=\tilde{s}_{i}\left(s_{j}, \varphi, \psi, \theta\right)=\sum_{i=-2}^{2} A_{i j} s_{j} .
$$

For the first one-parameter subgroup

$$
U=U_{\theta}=\left[\begin{array}{ccc}
\cos (\theta) & -\sin (\theta) & 0 \\
\sin (\theta) & \cos (\theta) & 0 \\
0 & 0 & 1
\end{array}\right]
$$

(around the axis $x_{-1}$ ) we obtain the following matrix

$$
A_{\theta}=\left[\begin{array}{ccccc}
\cos (\theta) & 0 & 0 & -\sin (\theta) & 0 \\
0 & \cos (2 \theta) & \frac{1}{2} \sqrt{3} \sin (2 \theta) & 0 & -\frac{1}{2} \sin (2 \theta) \\
0 & -\frac{1}{2} \sqrt{3} \sin (2 \theta) & \cos (\theta)^{2}-\frac{1}{2} \sin (\theta)^{2} & 0 & \frac{1}{2} \sqrt{3} \sin (\theta)^{2} \\
\sin (\theta) & 0 & 0 & \cos (\theta) & 0 \\
0 & \frac{1}{2} \sin (2 \theta) & \frac{1}{2} \sqrt{3} \sin (\theta)^{2} & 0 & \frac{1}{2}+\frac{1}{2} \cos (\theta)^{2}
\end{array}\right] .
$$

The two other subgroups are treated in a similar way. The knowledge of the obtained matrices will be helpful when considering different crystal systems below.

\subsection{Construction of invariants}

To write down all of the invariants of the variables $\left\{s_{i j}\right\}$ in (52), let us first ask, which quadratic forms (and how many) can be constructed from the elements $p$ and $\mathbf{s}$ from (55). For this purpose we write all possible Kronecker products of the weights 0 and 2

$$
0 \times 0,0 \times 2,2 \times 0,2 \times 2
$$

and their decompositions into irreducible ones. Note that, due to commutativity of the Kronecker product, the cases $0 \times 2$ and $2 \times 0$ are identical. According to (12), from the decomposition of the product of weights $N_{1}$ and $N_{2}$ into irreducible ones

$$
\left[2\left|N_{1}-N_{2}\right|+1\right]+\ldots+\left[2\left(N_{1}+N_{2}\right)+1\right]=\left(2 N_{1}+1\right)\left(2 N_{2}+1\right)
$$

parameters $\mathbf{w}_{n}^{(N)}$ arise, which transform by irreducible representations of the corresponding weights $N$. This fact is illustrated by the following formula [8]:

$$
\mathbf{p}^{\left(N_{1}\right)} \times \mathbf{q}^{\left(N_{2}\right)}=\sum_{N=\left|N_{1}-N_{2}\right|}^{N_{1}+N_{2}}\left(\sum_{n=-N}^{N} \mathbf{w}_{n}^{(N)} G_{N\left[N_{1}, N_{2}\right]}^{n}\right),
$$


where the vectors $\mathbf{p}$ and $\mathbf{q}$ are transformed by means of irreducible representations of weights $N_{1}$ and $N_{2}$, respectively.

The invariant quadratic forms (transformed by a representation of zero weight) made from these vectors look as follows (their invariance is an obvious consequence of orthogonality of the representations under consideration):

$$
\begin{aligned}
I_{(N)} & =\sum_{n=-N}^{N} \mathbf{w}_{n}^{(N)}\left(\left[\mathbf{p}^{\left(N_{1}\right)}\right]^{T} G_{N\left[N_{1}, N_{2}\right]}^{n}, \mathbf{q}^{\left(N_{2}\right)}\right), \\
N & =\left|N_{1}-N_{2}\right|,\left|N_{1}-N_{2}\right|+1, \ldots, N_{1}+N_{2} .
\end{aligned}
$$

In this way, we can calculate how many parameters (the coefficients of these quadratic forms) characterizing the elastic media there are and write down all possible invariant quadratic forms in (52). During this process we will identify the coinciding (due to commutativity) invariants and remove the skew-symmetric ones (for the odd sum of weights $N+N_{1}+N_{2}$ ). Finally we will obtain, according to 61],

1) $0 \times 0 \Longrightarrow 1$ parameter;

2) $0 \times 2,2 \times 0 \Longrightarrow 5$ parameters;

3) $2 \times 2 \Longrightarrow 1+5+9=15$ parameters (for the weights $0,2,4$; note that the cases of weights 1,3 are not meaningful because of the skew-symmetry of the korresponding Clebsch-Gordan matrices),

thus 21 parameters characterizing the elastic media, which is in accordance with the classical elasticity theory.

According to (63), the corresponding invariant quadratic forms look as follows.

1) For $N 1=0 ; N 2=0 ; N=0$ we have

$$
J_{0}=c_{1} G_{0[0,0]}^{0} p^{2}=c_{1} p^{2} .
$$

2) For $N 1=0 ; N 2=2 ; N=2$ the Clebsch-Gordan matrices look again very simple: $G_{2[0,2]}^{0}=\left[\begin{array}{lllll}0 & 0 & 1 & 0 & 0\end{array}\right], G_{2[0,2]}^{-1}=\left[\begin{array}{lllll}0 & 1 & 0 & 0 & 0\end{array}\right], G_{2[0,2]}^{1}=\left[\begin{array}{lllll}0 & 0 & 0 & 1 & 0\end{array}\right], G_{2[0,2]}^{-2}=$ $\left[\begin{array}{lllll}1 & 0 & 0 & 0 & 0\end{array}\right], G_{2[0,2}^{2}=\left[\begin{array}{lllll}0 & 0 & 0 & 0 & 1\end{array}\right]$, and the correspondinq quadratic form has five arbitrary parameters $a_{j}, j=-2,-1, \ldots, 2$ :

$$
J_{1}=\sum_{j=-2}^{2} a_{j} G_{2[0,2]}^{j} \mathbf{s} p=\sum_{j=-2}^{2} a_{j} s_{j} p .
$$

The same expression appears from the case $N 1=2 ; N 2=0 ; N=2$.

3) For the case $N 1=2 ; N 2=2$, as explained above, we have to consider $N=0,2,4$.

- For $N=0$ we have

$$
I_{0}=c_{2}\left(G_{0[2,2]}^{0} \mathbf{s}, \mathbf{s}\right)=\tilde{c}_{2}(\mathbf{s}, \mathbf{s}),
$$

where $G_{0[2,2]}=c I_{3}$ is a diagonal matrix.

- For $N 1=2 ; N 2=2 ; N=2$ the invariant quadratic form looks like

$$
I_{1}=\sum_{j=-2}^{2} b_{j}\left(G_{2[2,2]}^{j} \mathbf{s}, \mathbf{s}\right),
$$


where

$$
\begin{aligned}
& G_{2[2,2]}^{-2}=\left[\begin{array}{ccccc}
0 & 0 & -\frac{\sqrt{2}}{\sqrt{7}} & 0 & 0 \\
0 & 0 & 0 & -\frac{\sqrt{3}}{\sqrt{2} \sqrt{7}} & 0 \\
-\frac{\sqrt{2}}{\sqrt{7}} & 0 & 0 & 0 & 0 \\
0 & -\frac{\sqrt{3}}{\sqrt{2} \sqrt{7}} & 0 & 0 & 0 \\
0 & 0 & 0 & 0 & 0
\end{array}\right], \\
& G_{2[2,2]}^{-1}=\left[\begin{array}{ccccc}
0 & 0 & 0 & -\frac{\sqrt{3}}{\sqrt{2} \sqrt{7}} & 0 \\
0 & 0 & \frac{1}{\sqrt{2} \sqrt{7}} & 0 & \frac{\sqrt{3}}{\sqrt{2} \sqrt{7}} \\
0 & \frac{1}{\sqrt{2} \sqrt{7}} & 0 & 0 & 0 \\
-\frac{\sqrt{3}}{\sqrt{2} \sqrt{7}} & 0 & 0 & 0 & 0 \\
0 & \frac{\sqrt{3}}{\sqrt{2} \sqrt{7}} & 0 & 0 & 0
\end{array}\right] \text {, } \\
& G_{2[2,2]}^{0}=\left[\begin{array}{ccccc}
-\frac{\sqrt{2}}{\sqrt{7}} & 0 & 0 & 0 & 0 \\
0 & \frac{1}{\sqrt{2} \sqrt{7}} & 0 & 0 & 0 \\
0 & 0 & \frac{\sqrt{2}}{\sqrt{7}} & 0 & 0 \\
0 & 0 & 0 & \frac{1}{\sqrt{2} \sqrt{7}} & 0 \\
0 & 0 & 0 & 0 & -\frac{\sqrt{2}}{\sqrt{7}}
\end{array}\right] \\
& G_{2[2,2]}^{1}=\left[\begin{array}{ccccc}
0 & -\frac{\sqrt{3}}{\sqrt{2} \sqrt{7}} & 0 & 0 & 0 \\
-\frac{\sqrt{3}}{\sqrt{2} \sqrt{7}} & 0 & 0 & 0 & 0 \\
0 & 0 & 0 & \frac{1}{\sqrt{2} \sqrt{7}} & 0 \\
0 & 0 & \frac{1}{\sqrt{2} \sqrt{7}} & 0 & -\frac{\sqrt{3}}{\sqrt{2} \sqrt{7}} \\
0 & 0 & 0 & -\frac{\sqrt{3}}{\sqrt{2} \sqrt{7}} & 0
\end{array}\right] \text {, } \\
& G_{2[2,2]}^{2}=\left[\begin{array}{ccccc}
0 & 0 & 0 & 0 & 0 \\
0 & \frac{\sqrt{3}}{\sqrt{2} \sqrt{7}} & 0 & 0 & 0 \\
0 & 0 & 0 & 0 & -\frac{\sqrt{2}}{\sqrt{7}} \\
0 & 0 & 0 & -\frac{\sqrt{3}}{\sqrt{2} \sqrt{7}} & 0 \\
0 & 0 & -\frac{\sqrt{2}}{\sqrt{7}} & 0 & 0
\end{array}\right] \text {. }
\end{aligned}
$$

- Finally, for the case $N 1=2 ; N 2=2 ; N=4$ we obtain

$$
I_{2}=\sum_{j=-4}^{4} d_{j}\left(G_{4[2,2]}^{j} \mathbf{s}, \mathbf{s}\right),
$$

where

$$
\begin{gathered}
G_{4[2,2]}^{-4}=\left[\begin{array}{ccccc}
0 & 0 & 0 & 0 & -\frac{\sqrt{2}}{2} \\
0 & 0 & 0 & 0 & 0 \\
0 & 0 & 0 & 0 & 0 \\
0 & 0 & 0 & 0 & 0 \\
-\frac{\sqrt{2}}{2} & 0 & 0 & 0 & 0
\end{array}\right], G_{4[2,2]}^{-3}=\left[\begin{array}{ccccc}
0 & 0 & 0 & -\frac{1}{2} & 0 \\
0 & 0 & 0 & 0 & -\frac{1}{2} \\
0 & 0 & 0 & 0 & 0 \\
-\frac{1}{2} & 0 & 0 & 0 & 0 \\
0 & -\frac{1}{2} & 0 & 0 & 0
\end{array}\right], \\
G_{4[2,2]}^{-2}=\left[\begin{array}{ccccc}
0 & 0 & \frac{\sqrt{14} \sqrt{3}}{14} & 0 & 0 \\
0 & 0 & 0 & -\frac{\sqrt{14}}{7} & 0 \\
\frac{\sqrt{14} \sqrt{3}}{14} & 0 & 0 & 0 & 0 \\
0 & -\frac{\sqrt{14}}{7} & 0 & 0 & 0 \\
0 & 0 & 0 & 0 & 0
\end{array}\right],
\end{gathered}
$$




$$
\begin{gathered}
G_{4[2,2]}^{-1}=\left[\begin{array}{cccccc}
0 & 0 & 0 & \frac{\sqrt{7}}{14} & 0 \\
0 & 0 & \frac{\sqrt{7} \sqrt{3}}{7} & 0 & -\frac{\sqrt{7}}{14} \\
0 & \frac{\sqrt{7} \sqrt{3}}{7} & 0 & 0 & 0 \\
\frac{\sqrt{7}}{14} & 0 & 0 & 0 & 0 \\
0 & -\frac{\sqrt{7}}{14} & 0 & 0 & 0
\end{array}\right], \\
G_{4[2,2]}^{0}=\left[\begin{array}{ccccccc}
\frac{\sqrt{5} \sqrt{2} \sqrt{7}}{70} \\
0
\end{array}\right] \\
0 \\
0 \\
0
\end{gathered}
$$

As we can see, the 21 parameters characterizing the elastic media have split into several groups:

- two scalars $c_{1}, c_{2}$;

- two 5-dimensional vectors $\mathbf{a}=\left(a_{-2}, a_{-1}, a_{0}, a_{1}, a_{2}\right)$ and $\mathbf{b}=\left(b_{-2}, b_{-1}, b_{0}, b_{1}, b_{2}\right)$;

- one 9-dimensional vector $\mathbf{d}=\left(d_{-4}, d_{-3}, d_{-2}, d_{-1}, d_{0}, d_{1}, d_{2}, d_{3}, d_{4}\right)$.

\subsection{Examples}

For the crystal systems, where there are additional symmetries, the number of independent parameters is less than 21. Using again the representation group approach, this fact can be established easily for all of the seven crystal systems, also called syngonies. Below this is shown on the example of the monoclinic and rhombic crystal systems.

Recall that a crystal group $\Delta$ consists of several orthogonal transformations:

$$
\Delta \subset\left\{h: x \mapsto \tau+B x \mid \tau \in \mathbb{R}^{3}, B \in O(3)\right\},
$$

and $K$ is a crystal with the symmetry group $\Delta$, if

$$
K=h K, \forall h \in \Delta .
$$


Denote as $\Gamma$ the group of rotations of the crystal, i.e. the set of matrices constituting the elements of $\Delta$.

For the triclinic system, when $\Gamma=I$, we have the full set of 21 independent parameters. For the monoclinic system we have $\Gamma=<R_{2}>$, where

$$
R_{2}=\left[\begin{array}{ccc}
-1 & 0 & 0 \\
0 & -1 & 0 \\
0 & 0 & 1
\end{array}\right]=U_{\theta}
$$

is the rotation around the axis $x_{-1}$ on $\theta=\pi$. In this case the transformation matrix (60) looks like

$$
A=A_{\theta}=\left[\begin{array}{ccccc}
-1 & 0 & 0 & 0 & 0 \\
0 & 1 & 0 & 0 & 0 \\
0 & 0 & 1 & 0 & 0 \\
0 & 0 & 0 & -1 & 0 \\
0 & 0 & 0 & 0 & 1
\end{array}\right]
$$

Taking in a account the invariance of the quadratic forms introduced above, we arrive at the following relations: for the invariant $J_{1}$ from (65),

$$
\begin{gathered}
\sum_{j=-2}^{2} a_{j} s_{j}=a_{-2} s_{-2}+a_{-1} s_{-1}+a_{0} s_{0}+a_{1} s_{1}+a_{2} s_{2}= \\
=-a_{-2} s_{-2}+a_{-1} s_{-1}+a_{0} s_{0}-a_{1} s_{1}+a_{2} s_{2}=\sum_{j=-2}^{2} a_{j} \tilde{s}_{j},
\end{gathered}
$$

from where it follows that

$$
a_{-2}=a_{1}=0
$$

The identity for the invariant $I_{1}$ from (67), looking as

$$
\sum_{j=-2}^{2} b_{j}<G_{2[2,2]}^{j} \mathbf{s}, \mathbf{s}>=\sum_{j=-2}^{2} b_{j}<G_{2[2,2]}^{j} \tilde{\mathbf{s}}, \tilde{\mathbf{s}}>=\sum_{j=-2}^{2} b_{j}<A^{T} G_{2[2,2]}^{j} A \mathbf{s}, \mathbf{s}>
$$

gives

$$
b_{-2}=b_{1}=0 .
$$

Finally, the identity for the invariant $I_{2}$ from (68) we have

$$
\sum_{j=-4}^{4} d_{j}<G_{4[2,2]}^{j} \mathbf{s}, \mathbf{s}>=\sum_{j=-4}^{4} d_{j}<G_{4[2,2]}^{j} \tilde{\mathbf{s}}, \tilde{\mathbf{s}}>=\sum_{j=-4}^{4} d_{j}<A^{T} G_{4[2,2]}^{j} A \mathbf{s}, \mathbf{s}>
$$

from where

$$
d_{-4}=d_{-2}=d_{1}=d_{3}=0 .
$$

Consequently, for the monoclinic crystal system there are $21-2-2-4=13$ independent parameters which characterize the media. The quadratic invariants look in this case as

$$
\begin{gathered}
J_{0}=c_{1} \mathbf{p}^{2}, J_{1}=\left(a_{-1} G_{2[0,2]}^{-1}+a_{0} G_{2[0,2]}^{0}+a_{2} G_{2[0,2]}^{2}\right) \mathbf{s} p, \\
I_{0}=c_{2}(\mathbf{s}, \mathbf{s}), I_{1}=\left\langle\left(b_{-1} G_{2[2,2]}^{-1}+b_{0} G_{2[2,2]}^{0}+b_{2} G_{2[2,2]}^{2}\right) \mathbf{s}, \mathbf{s}\right\rangle, \\
I_{2}=\left\langle\left(d_{-3} G_{4[2,2]}^{-3}+d_{-1} G_{4[2,2]}^{-1}+d_{0} G_{4[2,2]}^{0}+d_{2} G_{4[2,2]}^{2}+d_{4} G_{4[2,2]}^{4}\right) \mathbf{s}, \mathbf{s}\right\rangle .
\end{gathered}
$$


For the rhombic system $<R_{2}, L_{2}>$, where

$$
L_{2}=\left[\begin{array}{ccc}
-1 & 0 & 0 \\
0 & 1 & 0 \\
0 & 0 & -1
\end{array}\right]=U_{\varphi}
$$

for $\varphi=\pi$, we need one more matrix, corresponding to the rotation $L_{2}$ :

$$
A=A_{\varphi}=\left[\begin{array}{ccccc}
1 & 0 & 0 & 0 & 0 \\
0 & -1 & 0 & 0 & 0 \\
0 & 0 & 1 & 0 & 0 \\
0 & 0 & 0 & -1 & 0 \\
0 & 0 & 0 & 0 & 1
\end{array}\right]
$$

Besides the zero coefficients obtained for the monoclinic system above, we have

$$
a_{-1}=b_{-1}=d_{-1}=d_{-3}=0 .
$$

The invariants look now like

$$
\begin{gathered}
J_{0}=c_{1} \mathbf{p}^{2}, J_{1}=a_{0} G_{2[0,2]}^{0}+a_{2} G_{2[0,2]}^{2} \mathbf{s} p, \\
I_{0}=c_{2}(\mathbf{s}, \mathbf{s}), I_{1}=\left\langle\left(b_{0} G_{2[2,2]}^{0}+b_{2} G_{2[2,2]}^{2}\right) \mathbf{s}, \mathbf{s}\right\rangle, \\
\left.I_{2}=\left\langle\left(d_{0} G_{4[2,2]}^{0}+d_{2} G_{4[2,2]}^{2}+d_{4} G_{4[2,2]}^{4}\right) \mathbf{s}, \mathbf{s}\right)\right\rangle .
\end{gathered}
$$

For the five other crystal systems the independent parameters are calculated in a similar way. By another method, using the contravariance of the Hook's tensor, this was done in [20].

For the isotropic case the only quadratic invariants are

$$
J_{0}=c_{1} \mathbf{p}^{2}, I_{0}=c_{2}(\mathbf{s}, \mathbf{s}) .
$$

Finally, let us consider the example of linear elasticity theory, when the equations can be written as follows:

$$
\left\{\begin{array}{l}
\rho \frac{\partial u_{i}}{\partial t}-\frac{\partial \sigma_{i j}}{\partial x_{j}}=0 \\
\frac{\partial \varepsilon_{i j}}{\partial t}-\frac{1}{2}\left(\frac{\partial u_{i}}{\partial x_{j}}+\frac{\partial u_{j}}{\partial x_{i}}\right)=0
\end{array}\right.
$$

where $i, j=1,2,3, u_{i}$ are the velocities, $\varepsilon_{i j}=\varepsilon_{j i}$ is the tensor of deformations and $\sigma_{i j}=\sigma_{j i}$ is the tensor of stresses. From the Hook's law $\varepsilon_{i j}=c^{i j k l} \sigma_{k l}$,

$$
\left\{\begin{array}{l}
\rho \frac{\partial u_{i}}{\partial t}-\frac{\partial \sigma_{i j}}{\partial x_{i}}=0, \\
c^{i j k l} \frac{\partial \sigma_{k l}}{\partial t}-\frac{1}{2}\left(\frac{\partial u_{i}}{\partial x_{j}}+\frac{\partial u_{j}}{\partial x_{i}}\right)=0 .
\end{array}\right.
$$

It is a symmetric hyperbolic system (in the sense of Friedrichs 3]), which may written, by means of Clebsch-Gordan matrices, in an invariant form (here $\mathbf{v}=\left(u_{-1}, u_{0}, u_{1}\right)$ is the velocity vector, $p$ is pressure, $\mathbf{s}$ is the 5 -dimensional vector defined in (55)): 


$$
\left\{\begin{array}{l}
A_{1} \frac{\partial}{\partial t} \mathbf{v}+\Delta_{-} \mathbf{s}+\Delta_{+} \mathbf{p}=0 \\
\hat{A} \frac{\partial}{\partial t}\left(\begin{array}{c}
\mathbf{p} \\
\mathbf{s}
\end{array}\right)+\left(\begin{array}{cc}
\Delta_{-} \mathbf{v}^{(\mathbf{1})} & 0 \\
0 & \Delta_{+} \mathbf{v}^{(\mathbf{1})}
\end{array}\right)=0
\end{array}\right.
$$

Here

$$
\Delta_{-} \mathbf{u}^{(\mathbf{L})}=c_{-}(L) \sum_{i=-1}^{1} \frac{\partial}{\partial x_{i}} G_{1[L-1, L]}^{i} \mathbf{u}^{(\mathbf{L})}, \quad \Delta_{+} \mathbf{u}^{(\mathbf{L})}=c_{+}(L) \sum_{i=-1}^{1} \frac{\partial}{\partial x_{i}} G_{1[L+1, L]}^{i} \mathbf{u}^{(\mathbf{L})}
$$

are invariant under rotations matrix differential operators. The first of them lowers the weight of the vector from $L$ to $L-1$ (an analog of div), the second one makes the weight bigger, from $L$ to $L+1$ (an analog of $\mathrm{grad}$ ). The matrix $\hat{A}$ looks as

$$
\left(\begin{array}{cc}
A_{0} & 0 \\
0 & A_{2}
\end{array}\right)+\left(\begin{array}{cc}
0 & \sum_{j=-2}^{2} a_{j} G_{2[0,2]}^{j} \\
\sum_{j=-2}^{2} a_{j} G_{2[2,0]}^{j} & \sum_{j=-2}^{2} b_{j} G_{2[2,2]}^{j}+\sum_{j=-4}^{4} d_{j} G_{4[2,2]}^{j}
\end{array}\right)
$$

Here

$$
A_{0}=\hat{c}_{1} G_{0[0,0]}^{0}=\hat{c}_{1}=\frac{1}{3 \lambda+2 \mu}, A_{2}=\hat{c}_{2} G_{0[2,2]}^{0}=\left(\begin{array}{ccccc}
\frac{1}{\mu} & & & & \\
& \frac{1}{\mu} & & & \\
& & \frac{1}{\mu} & & \\
& & \frac{1}{\mu} & \\
& & & \frac{1}{\mu}
\end{array}\right),
$$

where $\lambda$ and $\mu$ are the Lame coefficients In the isotropic case the constants $a_{i}, b_{i}$ and $d_{j}$ are equal to zero. For the triclinic system all of the 21 constants are present, while for other crystal systems some of them are equal to zero or are linearly dependent, as described above. In the case of nonlinear elasticity the systems (82) and (83) look more complicated: the matrix by $\frac{\partial}{\partial t}$ is constructed from second partial derivatives of the potential $H$ from (52), which has to be a convex function, and several other equations may be needed to add to the system, depending on the model $20,6,10,12$.

Acknowledgements. I thank S.K. Godunov for suggesting me this problematic and fruitful discussions and to V.M. Gordienko for a consultation on his papers.

\section{References}

[1] Alex A., Kalus M., Huckleberry, A., von Delft, J. A numerical algorithm for the explicit calculation of SU(N) and $\mathrm{SL}(\mathrm{N}, \mathrm{C})$ Clebsch-Gordan coefficients. Journal of Mathematical Physics, 52:2, 023507, 2011.

[2] Edmonds A. R. Angular Momentum in Quantum Mechanics. Princeton, New Jersey: Princeton University Press, 1957.

[3] Friedrichs K.O. Symmetric hyperbolic linear differential equations. Communication on Pure and Applied Mathematics, 7, 345-392, 1954. 
[4] Gelfand I. M., Milnos R. A., Shapiro E. Ya. Representations of the rotation group and the Lorentz group. M., 1958. (Russian)

[5] Gliske S., Klink W. H., Ton-That T. Algorithms for Computing U(N) Clebsch Gordan Coefficients. Acta Applicandae Mathematicae 95:1, 51-72, 2007.

[6] Godunov S.K. The equation of elasticity with dissipation as a nontrivial example of thermodynamically consistent hyperbolic equations. J. Hyperbolic Diff. Equat. , 1:4, 235-249, 2004.

[7] Godunov S.K., Gordienko V.M. The Clebsch-Gordan coefficients with respect to various bases for unitary and orthogonal representations of $S U(2)$ and $S O(3)$. Siberian Mathematical Journal, 45:3, 443-458, 2004.

[8] Godunov S.K., Gordienko V.M. Complicated structures of Galilean-invariant conservation laws. Applied Mechanics and Technichal Physics, 43:2, 175-189, 2002.

[9] Godunov S.K., Mikhailova T.Yu. Representations of the rotation group an spherical functions. Novosibirsk, "Nauchnaya kniga", 1998. (Russian)

[10] Godunov S. K., Peshkov I. M. Symmetric hyperbolic equations in the nonlinear elasticity theory. Zh. Vychisl. Mat. Mat. Fiz., 48:6, 1034-1055, 2008.

[11] Godunov S.K., Romenskii E. I. Elements of Continuum Mechanics and Conservation Laws, Novosibirsk, Nauchnaya Kniga Publishers, 1998. (Russian)

[12] Godunov S. K., Mikhailova T. Yu., Romenskii E. I. Systems of thermodynamically coordinated laws of conservation invariant under rotations. Siberian Mathematical Journal, 37:4, 690-705, 1996.

[13] Gordienko V.M. Matrix entries of real representations of the groups $O(3)$ and SO(3). Siberian Mathematical Journal 43:1, 51-63, 2002.

[14] Karasev V.P., Shelepin V.A. Finite differences, Clebsch-Gordan coefficients, and hypergeometric functions. Theoretical and Mathematical Physics, 17, 991-998, 1973.

[15] Landau L. D., Lifshitz E. M., Kosevich A. M., Pitaevskii L.P. Elasticity theory. Elsevier, 1986.

[16] Mikhailova T. Yu. Thermodynamically consistent conservation laws with unknowns of arbitrary weight Siberian Mathematical Journal, 38:3, 528-538, 1997.

[17] Miller W. Symmetry and separation of variables. Addison-Wesley, Reading, Massachusetts, 1977.

[18] Peshkov I. M. Numerical simulation of discontinuous solutions in nonlinear elasticity theory. Journal of applied mechanics and technical physics, 50:5, 858-865, 2009.

[19] Sedov L.I. A Course in Continuum Mechanics: Basic equations and analytical techniques. Wolters-Noordhoff, 1971.

[20] Selivanova S.V. Invariant recording of elasticity theory equations. Journal of Applied Mechanics and Technical Physics 49:5, 809-822, 2008.

[21] Shelepin L.A. Calculus of Clebsch-Gordan coefficients and its physical applications. Proceedings of the Lebedev Physical Institute 70, 1-114, New York, 1975.

[22] Steigmann D.J. Invariants of the Stretch Tensors and their Application to Finite Elasticity Theory. Mathematics and Mechanics of Solids 7:4, 393-404, 2002. 
[23] Sternberg S. Group theory and physics. Cambridge University press, 1994.

[24] Vilenkin N.Ya. Special functions and representation theory. M., "Nauka", 1965. (Russian) 